\title{
Manifestation of unusual size effects in granular thin films prepared by pulsed laser deposition
}

\author{
S. Sergeenkov a,b,*, L. Cichetto Jr. ${ }^{\mathrm{b}, c, \mathrm{~d}}$, J.C.C.A. Diaz ${ }^{\mathrm{b}}$, W.B. Bastos ${ }^{\mathrm{d}}$, E. Longo ${ }^{\mathrm{c}, \mathrm{d}}$, \\ F.M. Araújo-Moreira ${ }^{\mathrm{b}}$ \\ a Department of Physics, CCEN, Universidade Federal da Paraíba, 58051-970 João Pessoa, PB, Brazil \\ ${ }^{\mathrm{b}}$ Department of Physics, Universidade Federal de São Carlos, 13565-905 São Carlos, SP, Brazil \\ ${ }^{c}$ LIEC - Department of Chemistry, Universidade Federal de São Carlos, 13565-905 São Carlos, SP, Brazil \\ ${ }^{\mathrm{d}}$ Institute of Chemistry, Universidade Estadual Paulista - Unesp, 14801-907 Araraquara, SP, Brazil
}

\section{A R T I C L E I N F O}

\section{Article history:}

Received 21 January 2016

Received in revised form

2 May 2016

Accepted 3 June 2016

Available online 6 June 2016

Keywords:

Nickelates

Thin films

Pulsed laser deposition

Resistivity

Crossover

\begin{abstract}
A B S T R A C T
We demonstrate manifestation of some rather unusual size effects in granular thin films prepared by a pulsed laser deposition technique. We observed that the temperature dependence of resistivity $\rho(T)$ notably depends on the relation between the grain size $R_{g}$ and the film thickness $d$. Namely, more granular $\mathrm{LaNiO}_{3}$ thin films (with small values of $R_{\mathrm{g}}$ ) grown on $\mathrm{LaAlO}_{3}$ substrate are found to follow a universal $\rho(T) \propto T^{3 / 2}$ law for all the measured temperatures. While less granular thin films (with larger values of $R_{g}$ ), exhibit a more complicated behavior accompanied by a clear-cut crossover (around $T_{c r}=200 \mathrm{~K}$ ), from $\rho(T) \propto T^{3 / 2}$ (for $20 \mathrm{~K}<T<200 \mathrm{~K}$ ) to $\rho(T) \propto T$ (for $200 \mathrm{~K}<T<300 \mathrm{~K}$ ). The obtained results are attributed to manifestation of the finite temperature size effects (when an average grain size $R_{g}$ becomes comparable with the thermal de Broglie wavelength $\Lambda$ ) leading to the crossover temperature $T_{c r} \propto\left(d / R_{g}\right)^{2}$.
\end{abstract}

(c) 2016 Elsevier Ltd. All rights reserved.

\section{Introduction}

Recently, ferroelectric thin films have received much attention for their application in non-volatile random memory (NVRAM) and microsensors devices [1-4]. Due to improved fatigue and aging characteristics, various types of conducting oxides with perovskite crystal structure (applied as bottom electrodes) have been used, including $\mathrm{YBa}_{2} \mathrm{Cu}_{3} \mathrm{O}_{7-\delta}$ (YBCO) [5], $\mathrm{IrO}_{2}$ [6], $\mathrm{SrRuO}_{3}$ [7], and $\mathrm{LaNiO}_{3}$ (LNO) [8]. These materials possess noticeable advantages over the conventionally used Pt electrodes due to the fact that they better combine with ferroelectric thin films such as $(\mathrm{Pb}, \mathrm{Ca}, \mathrm{Sr}) \mathrm{TiO}_{3}[9], \mathrm{Pb}(\mathrm{Zr}, \mathrm{Ti}) \mathrm{O}_{3}[10], \mathrm{BaTiO}_{3}$ [11], etc. Probably one of the most promising materials for this purpose is the metallic oxide LNO [12-16] which has a rather good compatibility with many oxides substrates typically used for deposition, such as $\mathrm{SrTiO}_{3}$ (STO) and $\mathrm{LaAlO}_{3}$ (LAO), among others. When it comes to fabrication of LNO based thin films for their use as electrodes in lower layers for ferroelectric memory devices, it is of a vital importance to understand the underlying physical mechanisms governing the properties and, ultimately, the performance of these

\footnotetext{
* Corresponding author at: Department of Physics, Universidade Federal de São Carlos, 13565-905 São Carlos, SP, Brazil.

E-mail address: sergei@df.ufscar.br (S. Sergeenkov).
}

devices.

Clearly, the growing process plays the major role in obtaining high quality thin films fit for applications. In this regard, the pulsed laser deposition (PLD) technique is probably one of the best choices because it can provide samples with atomically smooth surfaces, matching the composition of the target, and providing controllable deposition at a wide range of oxygen pressure.

The prime goal of the present paper is to demonstrate how granularity of highly (100)-oriented LNO thin films deposited on LAO substrates by PLD influences the measured transport properties of these films. In particular, we found that the electron scattering mechanism (governing the temperature behavior of resistivity) drastically depends on the ratio between an average grain size $R_{g}$ and the film thickness $d$, providing a complimentary (to structural measurements) method to probe the films structure.

\section{Experimental}

In order to provide high quality samples, PLD technique was used to deposit thin films of LNO on (100) oriented LAO substrate with typical dimensions of $5 \times 5 \times 0.5 \mathrm{~mm}^{3}$. Laser wavelength and repetition rate were $\lambda=248 \mathrm{~nm}$ (KrF laser with $25 \mathrm{~ns}$ pulse duration) and $f=2 \mathrm{~Hz}$, respectively. The laser energy was maintained constant during the deposition and the beam was focused 
on the ceramic targets by a quartz lens to a fluency of around $1.2 \mathrm{~J} \mathrm{~cm}^{-2}$ for all the samples. During ablation, the target was rotated $(20 \mathrm{rpm})$ in order to reduce non uniform erosion and to get the films as homogeneous as possible. The heater power was monitored by a computer during the increase and decrease of the temperature. The temperature during deposition was measured by a thermocouple in contact with the heater and the bottom (back side) of the substrate. The substrate was placed parallel to the target and the distance between them was around $4.8 \mathrm{~cm}$ which was the best distance found due to the length of the plume. Before deposition, the base pressure of $P_{\text {base }} \leq 10^{-7} \mathrm{mbar}$ was applied and then the substrates were heated at $800^{\circ} \mathrm{C}$ for $20 \mathrm{~min}$ to get a carbon free and high crystalline surface. Deposition temperature for all films was defined as $T=625^{\circ} \mathrm{C}$ under a flowing oxygen pressure of $P_{\text {dep }}=0.22$ mbar maintained by a computerized mass flow controller to 80.0 SCCM (standard cubic centimetres per minute). After the deposition, the samples were in situ annealed at the same temperature of the deposition for $1 \mathrm{~h}$ under $500 \mathrm{mbar}$ oxygen pressure to improve the quality of films and decrease the oxygen vacancy. The dense and crack-free LNO ceramic circular target with diameter of $5 \mathrm{~cm}$ and thickness of $1.25 \mathrm{~cm}$ was prepared from highly pure polymeric precursors by Pechini method [17] using $\mathrm{La}_{2}\left(\mathrm{CO}_{3}\right)_{3} \times \mathrm{H}_{2} \mathrm{O} \quad(99.9 \% \quad$ Aldrich $)$ and $\mathrm{Ni}\left(\mathrm{OCOCH}_{3}\right)_{2} \times 4 \mathrm{H}_{2} \mathrm{O}(98 \%$ Aldrich $)$. The calcination and sintering were performed in the air at $900{ }^{\circ} \mathrm{C}$ for $4 \mathrm{~h}$ and at $1200^{\circ} \mathrm{C}$ for $6 \mathrm{~h}$, respectively. The target was polished after every film deposition to
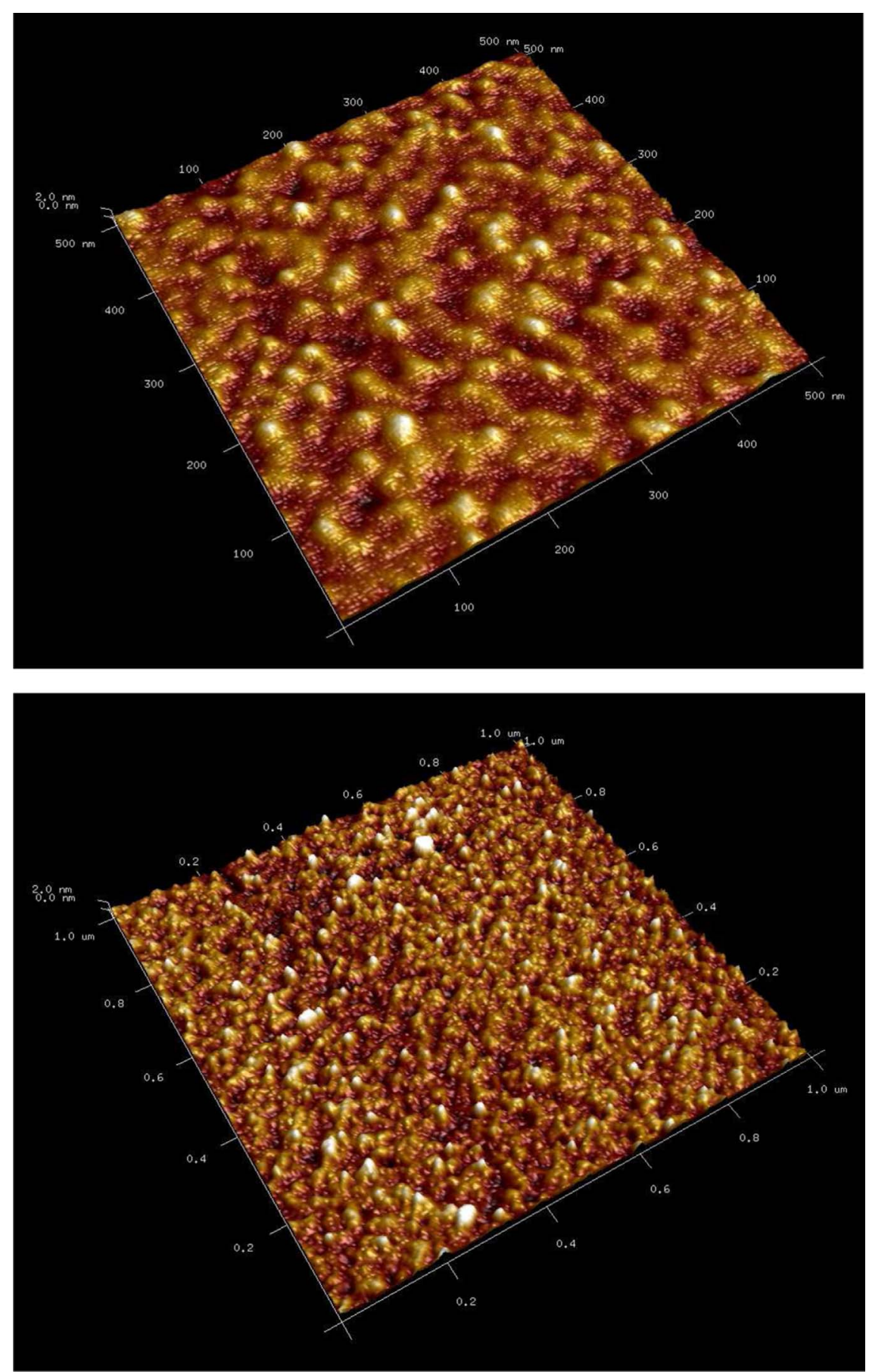

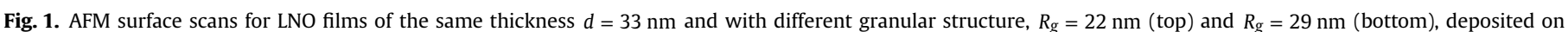
oriented LAO substrate. 
ensure comparable deposition conditions, especially the deposition rate. After that, the pre-ablation process was carried out for $30 \mathrm{~s}$ to prevent the deposition of the weakly bonded particles.

\section{Results and discussion}

To test the dependence of transport properties on the granularity of the sample, two different thin films (with the same thickness $d=33 \mathrm{~nm}$ ) have been prepared. The films thickness was confirmed by using field-emission scanning electron microscopy (FEG SEM) [13,18]. An average grain size of $R_{g}=22 \mathrm{~nm}$ and $R_{g}=29 \mathrm{~nm}$ for two studied films was deduced from the atomic force microscopy (AFM) images presented in Fig. 1.

It should be emphasized that the PLD technique is rather complicated and it involves many experimental parameters and conditions (such as surface heating, deposition rate, oxygen pressure, etc) which should be satisfied during the samples preparation and which are not so easy to control. One of the possible reasons for a markedly different granular structure could be attributed to slightly different deposition rates and/or a rather significant surface contamination from the environment (for one of the films). Further studies are needed to clarify this question.

Microstructure and crystallographic orientation of the films were characterized by X-ray diffraction (XRD) scans (see Fig. 2). XRD measurements of the films were carried out using a Shimadzu XRD-6000 diffractometer with $\mathrm{CuK} \alpha$ radiation. The unit cell refinements were performed using the Le Bail method through the GSAS/EXPGUI code $[19,20]$. The results revealed that our films crystallize into a cubic perovskite structure with a space group
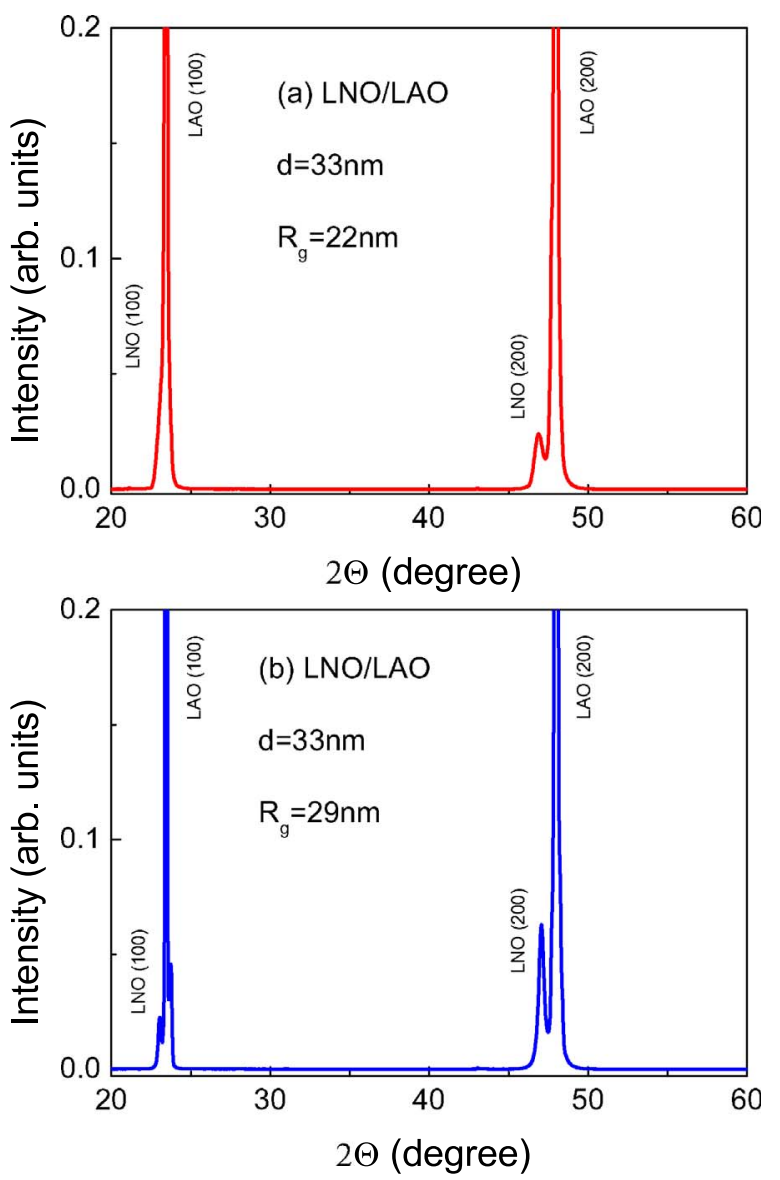

Fig. 2. XRD spectra of $L N O / L A O$ films of the same thickness $d=33 \mathrm{~nm}$ with different granular structure: (a) $R_{g}=22 \mathrm{~nm}$, and (b) $R_{g}=29 \mathrm{~nm}$.
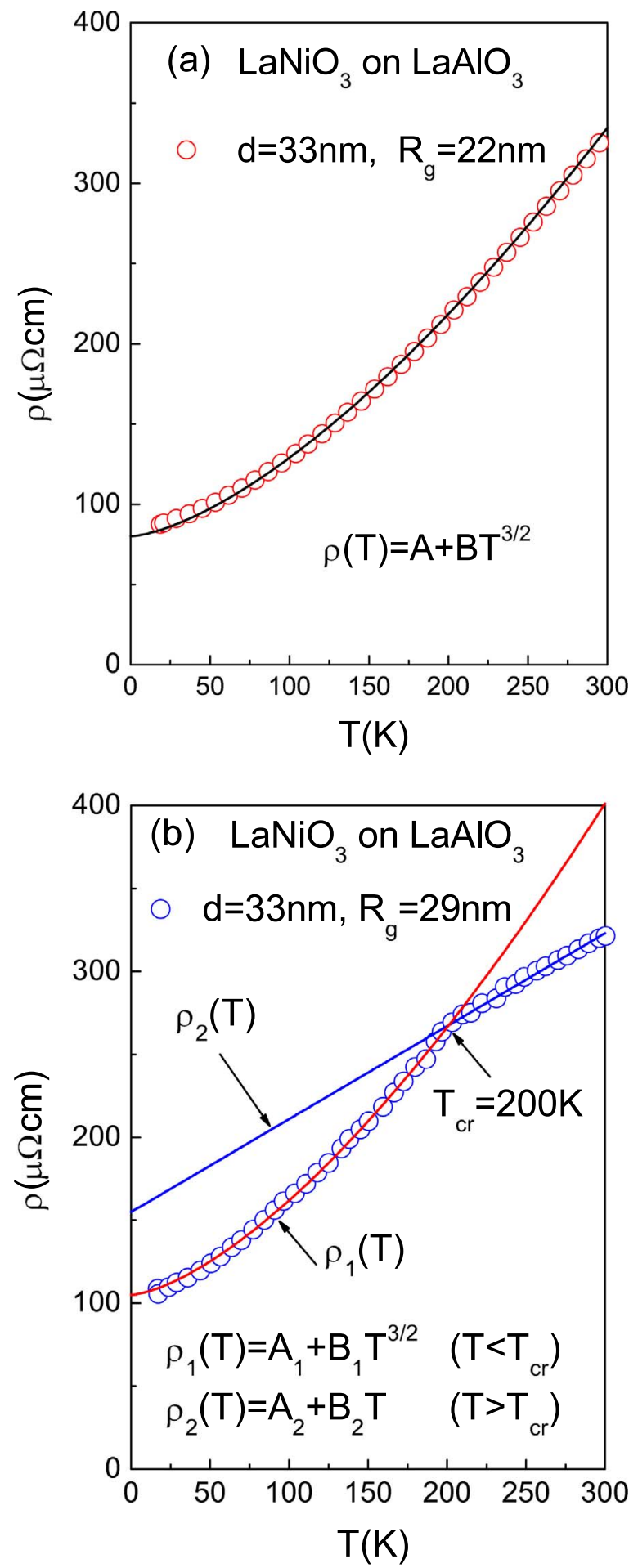

Fig. 3. Temperature dependence of the resistivity $\rho(T)$ measured for $L N O / L A O$ thin films of the same thickness $d=33 \mathrm{~nm}$ and two different grain sizes: (a) $R_{g}=22 \mathrm{~nm}$ and (b) $R_{g}=29 \mathrm{~nm}$. The solid lines are the best fits (see the text for details).

symmetry (221) $[\mathrm{Pm}-3 \mathrm{~m}]$ and lattice parameters around $a=0.386 \mathrm{~nm}$. Also, it was confirmed that the growth of the films occur in the (100) direction.

The electrical resistivity $\rho(T)$ was measured using the conventional four-probe method. To avoid Joule and Peltier effects, a dc current $I=100 \mu \mathrm{A}$ was injected (as a one second pulse) successively on both sides of the sample [21,22]. The voltage drop $V$ across the sample was measured with high accuracy by a KT256 nanovoltmeter. Fig. 3 shows the typical results for the temperature 
dependence of the resistivity $\rho(T)$ in our $L N O / L A O$ thin films heterostructures with different granularity. According to Fig. 3(a), a more granular film (with $R_{g} / d \simeq 0.6$ ) exhibits a universal $T^{3 / 2}$ behavior of resistivity over the entire measured temperature range and is well fitted by the following expression $[16,18]$ :

$\rho(T)=A+B T^{3 / 2}$

with $A=80 \mu \Omega \mathrm{cm}$ and $B=0.049 \mu \Omega \mathrm{cm} / \mathrm{K}^{3 / 2}$.

Recall $[16,18,23-27]$ that the $T^{3 / 2}$ law has been attributed to resonant scattering of conducting electrons on thermally activated spin fluctuations which begin to dominate the scattering process in our thin films heterostructure at the onset temperature $T_{s f} \simeq 23 \mathrm{~K}$.

On the other hand, according to Fig. 3(b), a less granular film (with $\quad R_{g} / d \simeq 0.9$ ) exhibits a clear-cut crossover (around $T_{c r}=200 \mathrm{~K}$ ) from the $\rho(T) \propto T^{3 / 2}$ behavior (for $20 \mathrm{~K}<T<200 \mathrm{~K}$ ) to a linear $\rho(T) \propto T$ dependence (for $200 \mathrm{~K}<T<300 \mathrm{~K}$ ). The latter is known [15] to be a signature of the electron-phonon scattering mechanism. More precisely, the temperature dependence of resistivity for the less granular films can be reasonably well fitted by the following expressions below and above the crossover temperature $T_{c r}$, respectively:

$\rho_{1}(T)=A_{1}+B_{1} T^{3 / 2}, \quad T \leq T_{c r}$

and

$\rho_{2}(T)=A_{2}+B_{2} T, \quad T \geq T_{c r}$

with $A_{1}=105 \mu \Omega \mathrm{cm}, B_{1}=0.57 \mu \Omega \mathrm{cm} / \mathrm{K}^{3 / 2}, A_{2}=155 \mu \Omega \mathrm{cm}$, and $B_{2}=0.56 \mu \Omega \mathrm{cm} / \mathrm{K}$.

Notice that, in agreement with Fig. 3(b), the onset temperature for spin fluctuations driven scattering mechanism is $T_{s f} \simeq 15 \mathrm{~K}$, markedly lower than for more granular films. Besides, from the high-temperature linear temperature dependence, we can estimate an effective electron-phonon coupling constant $\lambda$ according to the following expression [13]:

$\lambda=\frac{\hbar \omega_{p}^{2} \epsilon_{0}}{k_{B}}\left[\frac{d \rho}{d T}\right]$

Using [13] $\hbar \omega_{p} \simeq 1.1 \mathrm{eV}$ for plasmon frequency in LNO films, we obtain $\lambda \simeq 0.2$, in a reasonable agreement with available data on this parameter $[28,29]$.

Let us discuss now a possible origin of the crossover temperature $T_{c r}$. Based on our previous experience with nanogranular (bulk) graphite [30] and superconducting thin films [31], we were able to come up with the following explicit expression:

$T_{c r}=\frac{\hbar^{2}}{\pi k_{B} m^{*} a^{2}}\left(\frac{d}{R_{g}}\right)^{2}$

Here, $a$ is the lattice parameter, and $m^{*}$ is an effective carrier mass.

The origin of Eq. (5) is related to the so-called temperature dependent size effects (modified for thin films) which manifest themselves when an average grain size $R_{g}$ becomes comparable with the thermal de Broglie wavelength $\Lambda(T)=\sqrt{\hbar^{2} \mid \pi m^{*} k_{B} T}$. More precisely, the crossover temperature in thin granular films is defined by the following condition: $\Lambda\left(T_{c r}\right)=(a / d) R_{g}$ which readily leads to Eq. (5). Using the experimental parameters for our second film, $T_{c r}=200 \mathrm{~K}, a=0.386 \mathrm{~nm}, d=33 \mathrm{~nm}$ and $R_{g}=29 \mathrm{~nm}$, from Eq. (5) we obtain $m^{*} \simeq 9.9 m_{e}$ for an effective electron mass (in terms of the free mass $m_{e}$ ), in a rather good agreement with the estimates of this parameter reported in the literature [32,33]. On the other hand, the same Eq. (5) predicts $T_{c r} \simeq 350 \mathrm{~K}$ for a crossover temperature in our first (more granular) film with $R_{g}=22 \mathrm{~nm}$. This is the reason why the crossover does not manifest itself in these films for the measured temperature interval $(20 \mathrm{~K}<T<300 \mathrm{~K})$. Probably for the same reason $\left(T_{c r} \geq 350 \mathrm{~K}\right)$, no crossover was observed in a high quality $L N O / L A O$ heterostructure [34].

\section{Conclusions}

In summary, by using a pulsed laser deposition technique, we prepared two $\mathrm{LaNiO}_{3}$ thin films (grown on $\mathrm{LaAlO}_{3}$ substrate) with the same thickness $d$ but two distinctively different grain sizes $R_{g}$. By measuring the temperature dependence of resistivity $\rho(T)$, we observed that more granular films (with smaller $R_{g}$ ) follow a universal $T^{3 / 2}$ law (attributed to resonant scattering of conducting electrons on thermally activated spin fluctuations) for all the measured temperatures. While less granular thin films (with larger $R_{g}$ ), exhibit a crossover (around $T_{c r}=200 \mathrm{~K}$ ), from $\rho(T) \propto T^{3 / 2}$ law (for $20 \mathrm{~K}<T<200 \mathrm{~K}$ ) to electron-phonon dominated linear law $\rho(T) \propto T$ (for $200 \mathrm{~K}<T<300 \mathrm{~K}$ ). The obtained results suggest that a possibility to observe a crossover like behavior of resistivity strongly depends on the $\left(d / R_{g}\right)$ ratio in the studied granular thin films.

\section{Acknowledgments}

We are very grateful to H. Kamimura and R.C. Gouveia from NanO LaB for their help with resistivity measurements. We would like to thank LMA-IQ for allowing us to use FEG-SEM facilities. This work was financially supported by Brazilian agencies FAPESQ (DCR-PB), FAPESP and CNPq. We are very thankful to FAPESP (CEPID CDMF 2013/07296-2 and 2014/01371-5) for continuous support of our project on nickelates.

\section{References}

[1] R. Ramesh, D.G. Schlom, Science 296 (2002) 1975-1978.

[2] A. Bhardwaj, N.V. Burbure, G.S. Rohrer, J. Am. Ceram. Soc. 93 (2010) 4129-4135.

[3] Chengju Fu, Zhixiong Huang, Jie Li, Dongyun Guo, J. Electron. Mater. 39 (2010) 258-263.

[4] Jiaxuan Liao, Xubo Wei, Ziqiang Xu, Xiongbang Wei, Peng Wang, Mater. Chem. Phys. 135 (2012) 1030-1037.

[5] Li Kun, Wen Zheng, Wu Di, Zhai Haifa, Li Aidong, J. Phys. D: Appl. Phys. 46 (2013) 035308-035312.

[6] Nakamura Takashi, Nakao Yuichi, Kamisawa Akira, Takasu Hidemi, Jpn. J. Appl. Phys. 33 (1994) 5207-5212.

[7] Vilas Shelke, Dipanjan Mazumdar, G. Srinivasan, Arunava Gupta, J. Appl. Phys. 109 (2011) 07D914-07D916.

[8] Jun Ge, Xianlin Dong, Ying Chen, Fei Cao, Genshui Wang, Appl. Phys. Lett. 102 (2013) 142905-142912.

[9] D.S.L. Pontes, F.M. Pontes, A.J. Chiquito, E. Longo, Mater. Sci. Eng. B 185 (2014) 123-129.

[10] Jinrong Cheng, Liang He, Shengwen Yu, Zhongyan Meng, Appl. Phys. Lett. 88 (2006) 152906-152913.

[11] Aidong Li, Chuanzhen Ge, Peng Lü, Wu Di, Sibei Xiong, Naiben Ming, Appl. Phys. Lett. 70 (1997) 1616-1620.

[12] T. Yu, Y.F. Chen, Z.G. Liu, X.Y. Chen, L. Sun, N.B. Ming, L.J. Shi, Mater. Lett. 26 (1996) 73-77.

[13] D.S.L. Pontes, F.M. Pontes, Marcelo A. Pereira-da-Silva, O.M. Berengue, A. J. Chiquito, E. Longo, Ceram. Int. 39 (2013) 8025-8034.

[14] Mingwei Zhu, Philipp Komissinskiy, Aldin Radetinac, Mehran Vafaee, Zhanjie Wang, Lambert Alff, Appl. Phys. Lett. 103 (2013) 141902-141906.

[15] Mingwei Zhu, Philipp Komissinskiy, Aldin Radetinac, Zhanjie Wang, Lambert Alff, J. Appl. Phys. 117 (2015) 155306-155310.

[16] S. Sergeenkov, L. Cichetto Jr., E. Longo, F.M. Araújo-Moreira, JETP Lett. 102 (2015) 383-386.

[17] M. Pechini, US Patent no. 3.330.697, 1967

[18] S. Sergeenkov, L. Cichetto Jr., M. Zampieri, E. Longo, F.M. Araújo-Moreira, J. Phys.: Condens. Matter 27 (2015) 485307-485311.

[19] A.C. Larson, R.B. Von Dreele, General Structure Analysis System (GSAS), Los Alamos National Laboratory Report LAUR 86-748, 2000.

[20] B.H. Toby, J. Appl. Crystallogr. 34 (2001) 210-221.

[21] S. Sergeenkov, H. Bougrine, M. Ausloos, R. Cloots, JETP Lett. 69 (1999) 858-862. 
[22] S. Sergeenkov, C. Córdova, L. Cichetto Jr., O.F. de Lima, E. Longo, F.M. AraújoMoreira, J. Alloy. Compd. 667 (2016) 18-23.

[23] M. Ausloos, L. Hubert, S. Dorbolo, A. Gilabert, R. Cloots, Phys. Rev. B 66 (2002) 174436-174446.

[24] S. Sergeenkov, A.J.C. Lanfredi, F.M. Araujo-Moreira, JETP Lett. 85 (2007) $592-596$.

[25] Irfan Mansuri, Dinesh Varshney, J. Alloy. Compd. 513 (2012) 256-263.

[26] Dinesh Varshney, Irfan Mansuri, M.W. Shaikh, Y.K. Kuo, Mater. Res. Bull. 48 (2013) 4606-4612.

[27] M.W. Shaikh, Irfan Mansuri, M.A. Dar, Dinesh Varshney, Mater. Sci. Semicond. Process. 35 (2015) 10-16.

[28] L. Qiao, X. Bi, Acta Mater. 57 (2009) 4109-4115.
[29] S. Pal, B.K. Chaudhuri, S. Neeleshwar, Y.Y. Chen, H.D. Yang, J. Appl. Phys. 97 (2005) 0437071-0437079.

[30] S. Sergeenkov, N.S. Souza, C. Speglich, V.A.G. Rivera, C.A. Cardoso, H. Pardo, A W. Mombru, F.M. Araújo-Moreira, J. Appl. Phys. 106 (2009) 116101-116113.

[31] S. Sergeenkov, A.J.C. Lanfredi, F.M. Araújo-Moreira, J. Appl. Phys. 100 (2006) $123903-123913$.

[32] J.-S. Zhou, J.B. Goodenough, B. Dabrowski, P.W. Klamut, Z. Bukowski, Phys. Rev. Lett. 84 (2000) 526-529.

[33] K. Horiba, R. Eguchi, M. Taguchi, A. Chainani, A. Kikkawa, Y. Senba, H. Ohashi, S. Shin, Phys. Rev. B 76 (2007) 155104-155111.

[34] Yogesh Kumar, R.J. Choudhary, Abhinav Pratap Singh, G. Anjum, Ravi Kumar, J. Appl. Phys. 108 (2010) 083706-083712. 